\title{
IL CAPITALISMO MUNICIPALE
}

\author{
FRANCO OSCULATI $(*)$
}

Nota presentata dal m.e. Silvio Beretta

(Adunanza del 3 marzo 2011)

SUNTO. - Lo scritto prende in esame le principali ragioni che hanno condotto numerosi Enti locali a costituire società per azioni e altri organismi affidando loro la gestione di svariati servizi e attività precedentemente realizzati all'interno. Tali ragioni si legano in parte alla regolamentazione dei servizi pubblici locali e in parte all'intenzione di superare vincoli di natura finanziaria. Nella maggior parte dei casi, e specialmente nel campo delle cosiddette società strumentali, risultano ampie le differenze tra settore pubblico e settore privato nel raggiungimento dei benefici legati alla "societarizzazione", come in particolare quelli derivanti dalla responsabilità limitata, dall'eventualità del fallimento, dagli obiettivi di profitto ed efficienza. In genere, nel settore pubblico, tali benefici sono di minore importanza e di incerta praticabilità.

$* * *$

ABSTRACT. - The paper describes the main reasons that lead Local Government to create joint-stock companies for the provision of services and activities previously managed internally. These reasons are basically linked to regulation, as well as to financial and organizational issues. The paper argues that, for the most part of the cases, there are several differences between the public and the private sector as regards the achievement of the expected benefits form "corporatization", such as limited liability, bankruptcy, efficiency, profits, etc.. In fact, in the public sector these benefits appear substantially less important in comparison with the private sector.

(*) Professore ordinario di Economia pubblica; Dipartimento di Scienze politiche e sociali; Università degli Studi di Pavia. E-mail: osculati@unipv.it 
L'espressione "capitalismo municipale" allude alla proprietà di imprese e organismi vari riferibile ai Comuni (e ad altri Enti locali). I Comuni, soprattutto dell'Italia centrale e settentrionale, posseggono, per inveterata tradizione, varie aziende municipalizzate attive principalmente nei settori di acqua, gas, rifiuti e trasporti urbani. Negli ultimi anni, questi stessi Comuni e tanti altri hanno via via creato vari soggetti esterni alle normali strutture amministrative, mantenendone la proprietà totale $\mathrm{o}$ parziale e incaricandoli di operare in svariati campi diversi da quelli tradizionali. Le "partecipate" (a varia struttura giuridica) costituiscono ormai una forma ampia e ricorrente di intervento dell'operatore pubblico locale. Emerge però più di un dubbio se da tale fenomeno possano derivare effettivi miglioramenti nell'efficienza economica delle istituzioni e progressi nella stessa fluidità democratica del sistema.

\section{QUANTE SONO?}

Secondo i dati reperibili dai bilanci consuntivi approvati dai singoli enti nel 2009 e raccolti dal Ministero per la pubblica amministrazione esistono 2.365 consorzi e 4.741 società partecipate dalle Pubbliche amministrazioni con 24.713 rappresentanti negli organi di governo. I dati (consultabili sul sito del Ministero) riferiti all'anno prima erano 1.785 consorzi, 3.356 società e 19.870 persone presenti nei consigli di amministrazione.

Secondo l'indagine della Corte dei Conti su Comuni e Province (con integrazione di dati Unioncamere e con elaborazione di questionari raccolti fino al settembre 2009), nel periodo 2005-2008 si era in presenza di partecipazioni facenti capo a 5.928 enti in 5.860 organismi: dei quali $3.787(65 \%)$ società (per azioni, a responsabilità limitata, consortili e cooperative) e 2.073 (35\%) organismi diversi, quali consorzi, aziende speciali e altro. ${ }^{1}$ Anche la Corte registra una crescita: 4.992 soggetti partecipati nel 2005, 5.254 nel 2006 e 5.485 nel 2007. ${ }^{2}$

In questa sede riteniamo di restringere l'esame alle società e a

1 Corte dei Conti, 2010. Indagine sul fenomeno delle partecipazioni in società ed altri organismi da parte di Comuni e Province, Deliberazione 14/szaut/2010/frg, pp. 3-4.

2 Ibidem, p. 11. 
organismi tipo le fondazioni, le quali ultime, a dispetto delle loro caratteristiche formali, soprattutto nella sottospecie di "fondazioni di partecipazione" (un ibrido non codificato) sono ridotte a funzionare come società, suscitando qualche specifica e fondata preoccupazione da parte della Corte dei Conti:

"Si sta diffondendo negli enti il ricorso ... a fondazioni che, non dotate di sufficiente patrimonio, di frequente generano perdite, esito incongruo per tali meccanismi. La fondazione, per sua natura, dovrebbe utilizzare il reddito derivante dal patrimonio per lo svolgimento della sua ordinaria attività e proseguire la stessa sino a che non venga intaccato o, comunque, non si esaurisca il patrimonio. Il concetto di perdita gestionale da ripianare è estraneo alla nozione di fondazione poiché si tratta di un ente incentrato sul patrimonio e non sulla partecipazione di più soggetti, come avviene nell'associazione o nella società. Di fatto, la situazione è particolare poiché spesso la fondazione che dovrebbe ricevere contributi o erogazioni da parte dei soggetti che fruiscono dell'attività o del servizio reso, ha nel proprio patrimonio unicamente i beni immobili nei quali opera e lo svolgimento dell'attività presuppone necessariamente un intervento finanziario da parte dell'ente locale nel quale opera. Ente che, peraltro, ha, in numerosi casi, la possibilità di procedere alla nomina degli amministratori dell'ente morale o perlomeno di una parte degli stessi e obblighi di vigilanza, previsti dagli statuti. ...mentre il Comune può erogare specifici contributi ad una fondazione, non può accollarsi l'onere di ripiano di anno in anno (mediante la previsione di un generico contributo annuale) o anche occasionalmente le perdite gestionali della fondazione perché alle stesse deve essere in grado di far fronte la fondazione col suo patrimonio. Ove l'ente locale assuma l'impegno di far fronte alle perdite gestionali, sia mediante l'erogazione di generici contributi annuali che il formale ripiano occasionale di perdite, viene meno la natura di fondazione dell'organismo agevolato che, di fatto, si trasforma in ente strumentale del Comune, assumendo natura pubblica alla stessa stregua di un'azienda speciale o di un organismo societario". ${ }^{3}$

Delle partecipate comunali (nel periodo 2005 - 2007), 2.584 erano riferibili a Comuni sotto i 30.000 abitanti, 488 a Comuni tra i 30.000 e i 50.000 abitanti e 930 a Comuni sopra i 50.000 abitanti. $^{4}$

3 Corte dei Conti, 2010. Indagine conoscitiva sulla finanza locale, V Commissione bilancio Camera dei Deputati, 20 gennaio, p. 15.

4 Corte dei Conti, 2010. Indagine sul fenomeno delle partecipazioni in società ed altri organismi da parte di Comuni e Province, Deliberazione n.14/szaut/2010/frg, p. 6. 


\section{Alla base Del FenOmeno}

Le partecipazioni totali o parziali di enti pubblici in società appaiono assai numerose. Dunque, ci deve essere un'ampia copertura giuridica al fenomeno. Fatto salvo che "impresa" è diverso da "società" e che, però, la veste societaria è di gran lunga prevalente per la maggior parte delle imprese di qualche importanza, la radice giuridica della proliferazione delle società pubbliche, nella sua essenza, può essere indicata nella normativa sottesa alle sentenze della Corte Costituzionale 326/2008 e 148/2009, secondo le quali ad un'" attività amministrativa in forma privatistica", perfettamente lecita, va aggiunta un" "attività d'impresa di enti pubblici", altrettanto giuridicamente fondata. ${ }^{5} \mathrm{Si}$ tratta di ambiti e logiche diverse che difficilmente potranno coesistere nel medesimo soggetto imprenditoriale, ovvero nella medesima società.

L'attività d'impresa di pubbliche autorità, fenomeno non nuovo, è riferibile a campi diversi, purché si riscontri un interesse pubblico.

"L'intrapresa diventa pubblica, qualunque sia l'industria cui si riferisce, quando lo Stato ritiene di poter, meglio dei privati, tutelare certi interessi pubblici od ottenere una maggior somma di utilità pubblica collettiva. Questo è il concetto che si deve tener presente: in altre parole, un'intrapresa può diventar pubblica quando si raggiungono colla stessa certi fini pubblici che non potrebbero raggiungersi per mezzo dell'intrapresa privata" ${ }^{\circ}$ (Einaudi, 1916, p. 41).

Certamente attività d'impresa dello Stato fu quella delle Partecipazioni statali, le quali, almeno fino agli anni '70 del '900, costituirono un sistema importante e positivo, ampio e diversificato. La medesima locuzione "attività d'impresa” oggi è estensibile ai servizi pubblici, in particolare locali, che sono prodotti in mercati regolamentati (o da sottoporre a regolamentazione) nei quali soggetti imprenditoriali pubblici operano (o dovrebbero operare) su un piano di parità con soggetti privati. Delle imprese pubbliche attive nei servizi pubblici loca-

5 Torchia L., La responsabilità amministrativa per le società in partecipazione pubblica. Astrid, Roma, 2009.

6 Einaudi L., Corso di scienza delle finanze. Edizione della Rivista "La riforma sociale", Terza edizione, Torino, 1916, p. 41. 
li non potremo disinteressarci completamente in questa sede, ma esse non saranno l'oggetto principale della nostra analisi.

Maggiore attenzione sarà invece riservata all'" attività amministrativa in forma privatistica". Se dagli strumenti e dalle forme privatistici isoliamo prima l'impresa e quindi la società (la spa e la srl), subito ci imbattiamo in più di un dubbio sull'idoneità di tale soggetto e di tale costruzione giuridica a fungere quale strumento dell'amministrazione pubblica. Per quanto riguarda l'impresa, ricordo che secondo Coase l'impresa entra in campo, per operare nel mercato, quando i costi di transazione, cioè dei contratti, diventano troppo gravosi. ${ }^{7}$

Per quanto riguarda la foggia giuridica dell'impresa, come da manuale economico, ${ }^{8}$ i pregi dell'istituto societario sono:

a) la stessa personalità giuridica, che semplifica le relazioni contrattuali dell'impresa determinando una separazione tra proprietà e società. Ovvero: la personalità giuridica protegge le fortune (il benessere) degli azionisti da eventuali rovesci del proprio business e, all'inverso ma ugualmente importante, isola la sorte dell'impresa da possibili disavventure economiche o finanziarie degli azionisti;

b) la responsabilità limitata, che assiste l'intraprendenza imprenditoriale riducendo il rischio connesso con ogni singolo progetto d'investimento;

c) la circolazione delle quote, che permette, se del caso, di trasferire il controllo dell'impresa verso mani più idonee.

In ambiente pubblico o amministrativo queste premesse tengono fino a un certo punto. In particolare:

aa) quanto può essere lontana l'autorità pubblica di riferimento da una sua società quando questa si avvia al fallimento? Il fallimento è ritenuto dall'economia standard un mezzo importante per indurre efficienza, ma difficilmente potrà essere lasciata fallire una società pubblica incaricata, per esempio, di provvedere a qualche fase del processo di applicazione dei tributi. Questo dell'applicazione dei tributi è esempio che richiama direttamente ciò che intendiamo normalmente per attività amministrativa, cioè un'attività tipica e irrinunciabile dello Stato (nella sue varie

7 Coase R.H., The nature of the firm. Economica 4, 1937, pp. 386-395.

8 Per esempio, Ricketts M., The economics of business enterprise, Edward Elgar, Cheltenham UK, 2002, specialmente pp. 108-111. 
articolazioni decentrate, come da art.114 della Costituzione). Si può menzionare qualcosa di simile anche per settori meno legati alla potestà statale e ricadenti nell'area dei servizi pubblici, area che, come abbiamo già visto, è più consona all'attività d'impresa. L'esempio può essere quello della raccolta dei rifiuti urbani. Quale ente pubblico proprietario di una società addetta a tale servizio può accettarne il fallimento? $\mathrm{O}$, quanto meno, accettarne il fallimento se non dopo avere trovato una soluzione alternativa? Si noti che nel campo di diversi servizi pubblici il problema del fallimento si può porre anche nel caso di un concessionario privato. Se l'impresa concessionaria della raccolta e smaltimento dei rifiuti, dei trasporti urbani e interurbani, dell'erogazione dell'acqua e simili chiude e fallisce in modo più o meno inatteso, come reagisce l'autorità concedente?

bb) la responsabilità limitata è principio strettamente connesso al rischio che ci si assume investendo e operando in un determinato mercato. Siamo quindi nell'ambito dell'" attività d'impresa di enti pubblici". Ma nell'ambito dell'attività amministrativa? Sembra improbabile che un'autorità pubblica possa affidare ad una sua società la costruzione per esempio di un ponte senza accettare verso i terzi (i fornitori del manufatto) una responsabilità che vada anche oltre il capitale riversato inizialmente nella società. In altri termini e similmente al punto aa, mentre il bail out (salvataggio) della società partecipata nuoce all'efficienza (induce all'azzardo morale), come può un ente di governo non assumere in via sussidiaria i debiti delle controllate, quando queste sono, di fatto, uno strumento dell'attività amministrativa? Tra le società pubbliche, potrà mai darsi il caso della costituzione di una bad company? Non sarebbe una forma di ripudio del debito pubblico?

cc) ovviamente, riguardo la circolazione delle quote, come possono azioni riferite ad un'attività amministrativa essere poste sul mercato? Comunque, se le quote azionarie vengono vendute a privati si determina la privatizzazione e necessariamente cessa la funzione amministrativa. Tracce di questa possono forse persistere nel caso di privatizzazione parziale e di golden share.

Nella storia del nostro paese degli ultimi 20 anni, nella crescita delle società pubbliche territoriali (di Regioni ed Enti locali) soltanto in parte si può rintracciare la dicotomia tra attività d'impresa di enti pub- 
blici e attività amministrativa mediante società, anche se il fenomeno si è sviluppato lungo due direttrici, quella dei servizi pubblici locali (essenzialmente: acqua, gas, trasporti locali, igiene urbana) e quella relativa ad altri servizi o funzioni.

Il primo filone prende impulso dalla normativa sul ciclo integrato dell'acqua ("Legge Galli" del 1994) e sulla liberalizzazione degli altri servizi locali ("a carattere industriale" secondo la terminologia degli anni '90). All'interno di tale normativa sono d'obbligo la scomparsa delle gestioni in economia e la trasformazione delle municipalizzate in spa, cioè in una forma giuridica vista come mezzo per l'accorpamento delle gestioni, nonché come preludio alla privatizzazione.

Il secondo filone ottiene un via libera decisivo con la legge finanziaria per il 2002 e si alimenta degli incentivi distorti connessi con il patto di stabilità interno. La legge 448/2001 opera un'apertura alle "esternalizzazioni" che, alla lettera, dovrebbe corrispondere ad un robusto ridimensionamento del settore pubblico.

Con l'art. 28 si rinvia ad un regolamento per individuare "gli enti pubblici, le amministrazioni, le agenzie e gli altri organismi ai quali non siano affidati compiti di garanzia di diritti di rilevanza costituzionale, finanziati direttamente o indirettamente a carico del bilancio dello Stato o di altri enti pubblici, disponendone la trasformazione in società per azioni o in fondazioni di diritto privato, la fusione o l'accorpamento con enti od organismi che svolgono attività analoghe o complementari, ovvero la soppressione o messa in liquidazione" (comma 1). "La trasformazione ... è subordinata alla verifica che i servizi siano più proficuamente erogabili al di fuori del settore pubblico" (comma 5).

Con l'art. 29 "Le pubbliche amministrazioni ..., nonché gli enti finanziati direttamente o indirettamente a carico del bilancio dello Stato sono autorizzati, anche in deroga alle vigenti disposizioni, a: a) acquistare sul mercato i servizi, originariamente prodotti al proprio interno, a condizioni di ottenere conseguenti economie di gestione; $b$ ) costituire, nel rispetto della condizione di economicità di cui alla lett. $a$, soggetti di diritto privato ai quali affidare lo svolgimento di servizi, svolti in precedenza”.

Secondo il legislatore di inizio millennio la forma privatistica della spa è connessa con la riduzione della spesa pubblica e con l'aumento degli ambiti di mercato. C'è modo di ritenere, invece, che la spa (e varianti), usata a proposito e più spesso a sproposito, si sia rivelata un mezzo di occultamento delle reali dimensioni della spesa pubblica e di contenimento dell'area di mercato. In nome del mercato o, forse, ancor più in ragione di una diffidenza preconcetta nei confronti dello Stato (e 
sue varie articolazioni territoriali) si è privilegiato uno strumento tipico dell'economia privata, quale è la società, ma si è giunti a mettere in pericolo la principale condizione di funzionamento del mercato che è la concorrenza. E' un risultato paradossale, ma non sorprendente considerato come in Italia il mercato tout court si intrecci con il mercato politico e come la protezione e la rendita prevalgano nei fatti, oltre le propagandistiche enunciazioni di principio sulle virtù salvifiche del (mercato) privato. Come vedremo meglio successivamente, tale incoerente esito è stato favorito dal patto di stabilità interno.

\section{SOCIETÀ DI SERVIZI, SOCIETÀ STRUMENTALI E LE ALTRE}

Oltre la distinzione ricavabile dalle due sentenze della Corte Costituzionale citate, nel vasto mondo delle società pubbliche locali possono utilmente individuarsi alcune ulteriori classificazioni:

a) 1.a. società dedite ai servizi pubblici locali; 2.a. società strumentali; 3.a. altre società. Della distinzione tra le società di servizi pubblici locali (di interesse generale), o utility, e le altre si riscontra più di una traccia nella normativa. Non ci si imbatte, invece, in una netta definizione delle spa strumentali, sebbene nella legge, ancora sostanzialmente disattesa, con cui si intendeva limitare il fenomeno della "societarizzazione" a livello locale, si legga di "società ... costituite o partecipate dalle amministrazioni pubbliche regionali e locali per la produzione di beni e servizi strumentali all'attività di tali enti” (art. 13, c. 1, 1. 248/2006). Come nota la Corte dei Conti, la giurisprudenza ha chiarito che si tratta di beni e servizi erogati a diretto e immediato supporto di funzioni amministrative di natura pubblicistica e, quindi, di attività rivolta essenzialmente alla stazione appaltante e non al pubblico, come nel caso dei servizi pubblici locali. ${ }^{9}$ Esiste, infine, una terza residuale ed eterogenea categoria di società non riconducibili né ai servizi pubblici locali, né alla produzione di beni e servizi strumentali. Delle spa pubbliche locali conteggiate dalla Corte dei Conti, all'incirca il 60\% si occupa di servizi pubblici locali e il $40 \%$ delle attività delle altre due categorie.

9 Corte dei Conti, 2010. Indagine sul fenomeno delle partecipazioni in società ed altri organismi da parte di Comuni e Province, Deliberazione n.14/szaut/2010/frg. 
b) 1.b. società di cui si avvale il soggetto pubblico per svolgere attività prima prodotte all'interno della pubblica amministrazione; 2.b. società che derivano dalla trasformazione di preesistenti municipalizzate o aziende speciali; 3 .b. società cui il soggetto pubblico ricorre per svolgere attività prima realizzate soltanto da soggetti privati o, 4.b., non realizzate da alcuno. Le società del primo tipo, grosso modo, corrispondono alle strumentali, ma possono individuarsi anche nel caso di un servizio pubblico locale, come per esempio l'acquedotto, prima gestito in economia. Le società del terzo e quarto tipo dovrebbero corrispondere all'attività d'impresa di enti pubblici di cui alle sentenze della Corte Costituzionale prima citate. I casi 1.b e 2.b rientrano nel fenomeno della societarizzazione.

c) 1.c. spa finanziate unicamente, o quasi, dal contratto di servizio acceso con l'ente proprietario; 2.c. spa finanziate prevalentemente da tariffe pagate da utenti (famiglie e imprese) o da prezzi pagati da clienti (come nel caso delle farmacie). Le prime coincidono con le strumentali (2.a) e le seconde comprendono le spa di servizi pubblici locali (1.a) e le altre (3.a).

d) 1.d. società che operano in un settore o mercato regolamentato a livello nazionale; 2.d. società in settori non regolamentati a livello nazionale. Sebbene i sistemi di regolamentazione dei monopoli naturali presenti nei servizi pubblici locali siano carenti, assumiamo che i settori soggetti a regolamentazione siano acqua, energia elettrica, gas, rifiuti solidi, trasporti.

Le società strumentali, sebbene siano di importanza minore delle società di pubblici servizi, presentano alcune interessanti questioni, ad incominciare dalla definizione stessa. "Strumentale", infatti, rinvia alla ricerca delle finalità degli enti locali. Dato che, pur essendo intitolato "Servizi pubblici locali", non soccorre per la sua genericità l'art. 112 del Tuel (D.lgs 267/2000 e successive modificazioni), ${ }^{10}$ potrebbe essere utile riferirsi all'elencazione delle funzioni, anzitutto di quelle essenziali. A legislazione vigente, anche questa pista non conduce molto avanti. Infatti, per i Comuni - che sono il livello di governo di prima e ultima

10 "Gli enti locali, nell'ambito delle rispettive competenze, provvedono alla gestione dei servizi pubblici che abbiano per oggetto produzione di beni ed attività rivolte a realizzare fini sociali e a promuovere lo sviluppo economico e civile delle comunità locali”. 
istanza - l'art. 13 ("Funzioni") del Tuel pone giustamente soltanto un principio di larga autonomia: "Spettano al Comune tutte le funzioni amministrative che riguardano la popolazione ed il territorio comunale, precipuamente nei settori organici dei servizi alla persona e alla comunità, dell'assetto e utilizzazione del territorio e dello sviluppo economico, salvo quanto non sia espressamente attribuito ad altri soggetti dalla legge statale o regionale, secondo le rispettive competenze".

Relativamente alle Province il Tuel propone un elenco di funzioni più limitato e preciso, ma con il secondo comma dell'art.19 lascia aperto, per così dire, un certo effetto di trascinamento. ${ }^{11}$

\section{LE SPA PUBBLICHE SONO GIURIDICAMENTE COME LE ALTRE?}

A tutta prima sembrerebbe di sì, perché nell'ordinamento civile non esiste un diritto speciale riservato alle società partecipate da enti pubblici. ${ }^{12}$ Per la verità, dopo la riforma del 2003 (D.lgs 6/2003), nel codice civile ci si imbatte, o meglio imbatteva, negli artt. 2449 e 2450. Essi rafforzano i poteri di nomina di amministratori e sindaci per lo Stato e gli enti pubblici con partecipazioni (non totalitarie) in spa. ${ }^{13}$ Sennonché il 2450 è stato abrogato dal dl 10/2007 (art. 3), mentre il

11 "La Provincia, in collaborazione con i Comuni e sulla base di programmi da essa proposti, promuove e coordina attività, nonché realizza opere di rilevante interesse provinciale sia nel settore economico, produttivo, commerciale turistico, sia in quello sociale, culturale e sportivo".

12 Corte dei Conti, 2008. Stato dei controlli della Corte dei Conti sugli organismi partecipati dagli Enti locali. Deliberazione n. 13, p. 30.

13 Art. 2449. "Società con partecipazione dello Stato o di enti pubblici. 1. Se lo Stato o gli enti pubblici hanno partecipazioni in una società per azioni, lo statuto può ad essi conferire la facoltà di nominare uno o più amministratori o sindaci ovvero componenti il consiglio di sorveglianza, 2. Gli amministratori e i sindaci o i componenti del consiglio di sorveglianza nominati a norma del comma precedente possono essere revocati soltanto dagli enti che li hanno nominati. 3. Essi hanno i diritti e gli obblighi dei membri nominati dall'assemblea. Sono salve le disposizioni delle leggi speciali. Art. 2450. Amministratori e sindaci nominati dallo Stato o da enti pubblici. 1. Le disposizioni dell'articolo precedente si applicano anche nel caso in cui la legge o lo statuto attribuisca allo Stato o a enti pubblici, anche in mancanza di partecipazione azionaria, la nomina di uno o più amministratori o sindaci o componenti del consiglio di sorveglianza, salvo che la legge disponga diversamente. 2. Qualora uno o più sindaci siano nominati dallo Stato, il presidente del consiglio sindacale deve essere scelto tra essi”. 
2449 è stato svuotato di significato in sede europea da una pronuncia della Corte di Giustizia (C-464/04 del 6/12/2007) che lo ha giudicato in contrasto con l'art. 56 del Trattato Ue, articolo riguardante la libera circolazione dei capitali.

Tuttavia, la natura del socio pubblico non può non influire, tanto più che il fenomeno del "capitalismo municipale", come visto al par. 1, è in espansione. Si sono quindi succeduti interventi legislativi sugli spazi di azione formalmente consentita alle spa pubbliche locali e su alcuni moduli organizzativi.

\section{A TUTELA DELLA CONCORRENZA}

Già nell'estate del 2006 si è provveduto a restringere l'offerta di beni e servizi della spa al solo ente locale costituente o partecipante (art. 13, legge 248/2006). In quel caso il riferimento specifico è stato alle società strumentali, ma il senso della norma ricalca uno dei requisiti fondamentali dell' in house possibile anche per i servizi pubblici locali. Le società, che si fossero trovate a fornire beni e servizi anche a enti diversi, avrebbero dovuto rinunciare entro dodici mesi, eventualmente scorporando in una separata società da cedere entro ulteriori diciotto mesi. L'efficacia di tali regole era assistita dalla previsione di annullamento dei contratti verso enti terzi, allo scadere del primo termine di dodici mesi. Non mi è noto se esistano indagini sugli effetti di questa parte della normativa. Certo, dal solo numero di spa locali, in aumento $\mathrm{o}$ in diminuzione, non è possibile inferire alcunché, in quanto, per esempio, se la spa dell'ente $A$ vendeva servizi al limitrofo ente $B$, può darsi che questo, proprio sulla spinta della legge, abbia deciso di dare vita alla propria società dei servizi strumentali prima acquistati presso la società di A (o di acquistare parte della società scorporata). Dall'intervento legislativo del 2006, comunque, ci si può aspettare più un aiuto al mantenimento della concorrenzialità dei mercati che una riduzione dello spazio del mercato o, nello specifico, della quantità delle commesse pubbliche destinate a operatori privati.

Infatti, sarebbe utile individuare fino a che punto la società strumentale o in house incrementa la produzione pubblica di beni e servizi in sostituzione di operatori privati. Può avvenire che alla spa venga né più né meno affidato il compito di curare i rapporti già in essere con fornitori privati. In questo caso, chi teme un restringimento dell'econo- 
mia privata non ha ragione di dolersi della societarizzazione. Al contrario, chi tiene alla trasparente gestione della cosa pubblica ha qualche motivo di preoccupazione.

Ad ogni buon conto, la normativa successiva contenuta nella finanziaria per il 2008 avrebbe dovuto determinare almeno un rallentamento del ritmo di creazione di spa pubbliche locali e di partecipazioni. Fatti salvi i settori dei servizi pubblici di interesse generale, si vietavano società e partecipazioni anche di minoranza e indirette "non strettamente necessarie per il perseguimento delle ... finalità istituzionali" degli enti. Società e partecipazioni senza i requisiti richiesti avrebbero dovuto essere dismesse entro il luglio 2009 (art. 3, cc. 27 e 29, 1. 244/2007). E' evidente, come già indicato al paragrafo precedente, che la definizione delle finalità istituzionali e la pertinenza di società e partecipazioni a queste, in concreto, sono lasciate alla buona volontà delle amministrazioni.

\section{SOCIETARIZZAZIONE E PICCOLI COMUNI}

Una norma della manovra dell'estate 2009 proibisce ai Comuni al di sotto dei 30.000 abitanti di costituire società e intima di procedere entro il 31 dicembre 2011 alla liquidazione delle spa o alla cessione delle partecipazioni in essere (a fine maggio 2010, data di entrata in vigore del dl 78). La norma (art. 14, c. 32 della 1. 122/2010, di conversione del 78) non si applica sicuramente: ${ }^{.4}$ a) a spa e partecipazioni riguardanti servizi di interesse generale (dato il richiamo all'art. 3, c. 27 , ultima parte, della 1. 244/2007); b) alle società "con partecipazione paritaria ovvero con partecipazione proporzionale al numero degli abitanti, costituite da più Comuni la cui popolazione complessiva superi i 30.000 abitanti". Sembra chiaro che, con questa specificazione, il legislatore riconosca l'utilità delle spa formate dai piccoli Comuni, approssimativamente a fini di attenuazione del sottodimensionamento e concretamente anche al fine di consentire la produzione di servizi impossibili da erogare se non oltre una certa soglia dimensionale.

14 Sul reale significato del c. 32 un'incertezza di fondo nasce all'incipit: "Fermo restando quanto previsto dall'art. 3, cc. 27, 28 e 29 della legge 24 dicembre 2007, n. 244, i Comuni con popolazione inferiore a 30.000 abitanti non possono costituire società". La legge 244 vietava esplicitamente soltanto le spa strumentali in senso stretto. 
E' invece ragionevole mostrarsi avversi alle società di un solo piccolo Comune o di soli piccoli o piccolissimi Comuni che, anche operando insieme, non raggiungono una soglia critica, perché il make all'interno della pubblica amministrazione, invece del buy all'esterno presso operatori privati o anche presso Comuni o società pubbliche di grandi dimensioni, può impedire di sfruttare le economie di scala. Successivamente, tuttavia, il legislatore rinuncia ad intervenire in questa materia. Infatti, con il "decreto Milleproroghe" del 2011 il termine del dicembre 2011 viene sostituito dal dicembre 2013 e, soprattutto, si limita l'obbligo della liquidazione o della cessione alle società senza utili per i tre anni precedenti il 2013 o con riduzioni di capitale o con perdite di bilancio ripianate dal Comune proprietario (l. 10/2011, art. 2, c. 43).

Riguardo ai Comuni di taglia demografica compresa tra i 30.000 e i 50.000 abitanti la manovra 2010 stabilisce il principio della partecipazione in una sola società. Nel caso siano più di una, il Comune dovrà disfarsene sempre entro il 31 dicembre 2011. Questi Comuni dovrebbero dunque avviare un processo di fusione tra la pluralità di società esistenti. Si dovrebbe trattare, è auspicabile, di fusioni effettive e non di holding. L'indicazione del dicembre 2011 si pone sostanzialmente come la proroga di un termine già abbondantemente scaduto. Come ricordato sopra, tutti i Comuni avrebbero dovuto disfarsi delle partecipazioni in spa non strettamente strumentali e non di servizi di interesse generale entro il luglio 2009 (art. 3, comma 29, 1. 244/2007).

Nel complesso, mentre mancano per il momento conferme di un qualche rispetto delle varie norme sulle dismissioni e cessioni, gli interventi legislativi del 2010 e del 2011, contrariamente alle attese espresse anche in sede molto autorevole, ${ }^{15}$ suonano come resa del tentativo di limitare il numero delle partecipate. Si può sperare che il tema venga ripreso e meglio inquadrato in sede di discussione parlamentare e di approvazione della "Carta delle autonomie". Per il momento, comunque, la pubblica amministrazione locale italiana continua ad alimentare

15 "Con la manovra finanziaria per il 2010 (dl 78/2010, art. 14, c. 32) sono stati imposti vincoli molto stringenti alle partecipazioni societarie dei Comuni: ne dovrebbe risultare ridimensionato il fenomeno presso gli enti di maggiori dimensioni e sostanzialmente eliminato presso quelli piccoli e medio-piccoli" (Corte dei Conti, Relazione sull'attività svolta nel 2010-2011, p. 52). 
l'impressione di volersi servire delle società come mezzo ordinario per produrre qualsivoglia utilità diretta o indiretta dell'ente proprietario.

\section{PATTO DI STABILITÀ E ALTRI VINCOLI}

Dunque, la forma giuridica societaria è tipico strumento del mercato, il quale è libero sia di inserire in tale contenitore qualunque contenuto lecito, sia di replicarne il numero. $\mathrm{Ma}$, se adottata dalla pubblica amministrazione locale, la veste societaria richiede (nel caso italiano ha richiesto) interventi legislativi a tutela della concorrenza e a disincentivo alla sua proliferazione. La normativa tendente a questi due scopi si è aggiunta alla regolamentazione resa necessaria dalla presenza di monopoli naturali riscontrabile nel settore dei servizi pubblici locali e ai vincoli di "servizio pubblico generale" necessariamente imposti al medesimo settore.

A tutto ciò, soprattutto negli ultimi tempi, si sono sovrapposte norme che entrano nel merito stesso dei moduli organizzativi delle società pubbliche locali. La dipendenza che molte tra queste vivono da fonti di finanziamento di tipo tributario (o di tipo debito pubblico) impedisce di considerarle estranee alla manovra, agli obiettivi e ai vincoli di finanza pubblica. Sebbene con un certo ritardo, le società pubbliche, quindi, sono state inserite tra gli oggetti del patto di stabilità interno. Il trapianto di regole pubblicistiche in ambito privatistico non sempre risulta facile.

"sembra piuttosto chiaro come il legislatore non appaia preoccupato di mantenere una adeguata coerenza delle disposizioni normative con le caratteristiche ontologiche del modello societario, quanto piuttosto di scongiurare, almeno teoricamente, il rischio che utilizzando strumentalmente specifici modelli organizzativi il socio pubblico possa porre in essere manovre elusive dei vincoli di finanza pubblica o violare principi giuridici fondamentali, anche di ordine costituzionale, tutelati da precise norme di garanzia. E a ciò ha proceduto senza valutare del tutto né la reale applicabilità né gli effetti su soggetti e attività di diritto comune di norme conformate a pubbliche amministrazioni”. ${ }^{16}$

16 Corte dei Conti, 2010. Indagine sul fenomeno delle partecipazioni in società ed altri organismi da parte di Comuni e Province, Deliberazione n.14/szaut/2010/frg, pp. 37-38. 
L'attività e gli stessi moduli organizzativi delle società pubbliche locali soggiaciono attualmente ad una serie di vincoli, prodotti per estensione dalla normativa sulla pubblica amministrazione normale o tipica, assunti principalmente in vista delle compatibilità macrofinanziarie (indebitamento netto della pubblica amministrazione e debito pubblico). E' in quest'ottica, infatti, che vengono assoggettate al patto di stabilità interno le società in house affidatarie di servizi pubblici locali ai sensi dell'art. 23 bis (c. 10, lett. a, legge 133/2008, anche come modificato dal dpr 135/2009, convertito dalla legge 166/2009). L'estensione del patto è contenuta anche nel dpr 168/2010 (art. 5) che è di attuazione del 23 bis e che è specifico del settore dei servizi pubblici locali. L'estensione, logicamente, dovrebbe valere per tutte le in bouse e, a maggior ragione, per i settori che vedono impegnate le società strumentali. In caso esistano in bouse consortili in proprietà anche di Comuni non tenuti al patto (attualmente i Comuni con meno di 5.000 abitanti), l'assoggettamento al patto opererebbe anche nei confronti di questi seppure limitatamente e per il tramite delle partecipate.

La recente (novembre 2010) sentenza 325 della Corte Costituzionale sembra modificare il quadro sin qui tracciato perché dichiara illegittima la soggezione al patto delle in house di servizi pubblici locali. La motivazione, tuttavia, può lasciare spazio a una riedizione del patto stesso operata in una veste diversa da quella costituita dalla legge 133/2008 e successive modificazioni. Infatti, la motivazione della Corte si appunta sulla ripartizione costituzionale delle competenze (art. 117 della Costituzione) e concerne più la procedura che il contenuto. Il patto attiene alla materia del coordinamento della finanza pubblica che è di competenza legislativa concorrente. Invece, la legge 133 riguarda una materia di esclusiva competenza legislativa statale, quale la tutela della concorrenza. Tale legge, inserendo una disposizione sul patto, ha per così dire sconfinato dal suo campo di pertinenza. Ne dovrebbe conseguire che, rispettando le adeguate procedure e competenze (non semplice "parere" della o "sentita" la, ma "d'intesa" con la Conferenza unificata), il patto potrà essere riproposto.

Il medesimo dpr 168 (art. 6), inoltre, richiede alle società in bouse, ma anche alle miste, di applicare il "Codice dei contratti pubblici relativi a lavori, servizi e forniture" (D.lgs 163/2006) e (all'art. 7), infine, impone, alle in bouse e alle miste di adottare per il reclutamento del personale e per il conferimento degli incarichi i medesimi criteri concorsuali previsti per l'impiego pubblico (art. 35, c. 3, D.lgs 165/2001). 
A differenza di quanto deciso sulla materia del patto di stabilità, la sentenza 325 della Corte costituzionale ha considerato legittime le norme sugli acquisti e sul personale previste dal 23 bis (c. 10, lettera $a$ ), di cui il dpr 168 è derivazione.

Le società in house, si rammenti, sono "a controllo analogo". L'espressione, se ha un senso, vuol dire che l'attività della spa è tale e quale quella di un assessorato ma se l'assessorato e l'intero ente cui esso partecipa sono sottoposti ai vincoli richiesti dall'equilibrio della finanza pubblica, è arduo sostenere che l'in house ne è esentata. Compreso il patto.

\section{PeRChÉ FARLE?}

Abbiamo visto che le società pubbliche locali sono state create in gran copia soprattutto perché (a) richieste dalla normativa sui servizi tipici erogati dalle precedenti municipalizzate e perché (b) utili come arma diversiva avversa al patto di stabilità interno. Ora, con un approccio di tipo normativo, occorre chiedersi quali sono o dovrebbero essere le finalità razionali, o teoriche, della societarizzazione, oltre al motivo scontato della trasformazione in spa come passo prodromico alla privatizzazione.

\subsection{Un primo argomento}

Un primo argomento potrebbe essere il seguente: nella spa, per effetto della specifica tutela giuridica degli azionisti, c'è rigore e non si spreca. A tacer d'altro, pur così impostato il tema non può trascurare le questioni cosiddette di agenzia (fino a che punto i manager sono aperti e leali nei confronti dei proprietari?) e, ancor più ampiamente, l'interrogativo sulle motivazioni dei manager. Questi devono operare nell'interesse della proprietà o nell'interesse della società? Simili quesiti nell'economia privata si impongono con indubbia gravità. Valgono, probabilmente, anche in tutti i casi di società pubblica di servizi ma applicati alle spa strumentali perdono di spessore. Chiedere se l'amministratore delegato di una spa incaricata dell'applicazione dei tributi locali tenga più alla società che al Comune proprietario non è diverso dal domandare se l'assessore alle finanze è più preoccupato dei destini dell'assessorato o del futuro dell'intero Comune. In entrambe le ipote- 
si sembra decisivo il rapporto che lega chi decide la nomina, per semplificare il sindaco, con l'amministratore delegato o con l'assessore.

Questo tipo di considerazioni era rafforzato dal fatto che la giurisdizione nei confronti di società di diritto privato partecipata da soggetto pubblico sembrava pacificamente assegnata alla magistratura contabile, cioè alla Corte dei Conti. Nel corso del 2010, invece, sentenze della Corte di Cassazione hanno precisato che il manager di società pubblica non è soggetto alla magistratura contabile ma alla magistratura ordinaria, la quale però deve essere adita dall'amministratore/proprietario pubblico in caso di danno patrimoniale.

“... la giurisdizione contabile si esercita unicamente sul danno inferto direttamente al patrimonio del soggetto pubblico partecipante (ad esempio: danno all'immagine), ma non per i danni che si assumono causati al patrimonio della società privata. Per questi ultimi è il socio pubblico, nella sussistenza dei presupposti di legge, ... ad esercitare innanzi al giudice ordinario l'azione sociale civile, nonché a poter essere chiamato a rispondere, innanzi al giudice contabile, di eventuali omissioni al riguardo, causative di nocumento erariale.

Non si può dire, quindi, che sia stata smentita la sussistenza della giurisdizione contabile ogniqualvolta la prospettazione della pretesa risarcitoria ... venga saldamente basata su censure concernenti un'attività svolta anche da soggetto totalmente privato, ma concretizzatasi in una "gestione pubblica":

- che sia esplicitamente funzionalizzata al perseguimento di finalità pubbliche;

- che abbia reso il soggetto privato compartecipe diretto e fattivo di attività istituzionali pubbliche; che abbia inferto danno ingiusto al patrimonio di una pubblica amministrazione". ${ }^{17}$

Difficile concludere, a questo punto, che l'assetto della giurisdizione sia stabile e, soprattutto, che l'innovazione abbia portato a una maggiore separazione tra proprietà pubblica e spa e che, per questa via, sia facilitata da maggiore autonomia e gravata da maggiore responsabilità. Un aspetto che andrebbe chiarito è quello dei tempi. Un sistema di doppia giurisdizione, ordinaria e contabile, probabilmente li allunga, se non altro perché la giurisdizione contabile, per poter intervenire in caso "di eventuali omissioni” di amministratori pubblici nei confronti di manager,

17 Corte dei Conti, 2011. Cerimonia di inaugurazione dell'anno giudiziario 2011. Relazione del Procuratore generale Mario Ristuccia. p. 12. 
deve attendere la scadenza dei termini della procedura civile. Se per ogni tipo di giustizia i tempi sono importanti, nella pubblica amministrazione possono essere decisivi perché incidono sui cicli elettorali.

I rapporti tra manager e amministrazione proprietaria dovrebbero, in particolare, essere regolati dall'art. 2497 del codice civile, relativo alla responsabilità per la corretta gestione societaria e imprenditoriale di società soggette alla direzione e coordinamento di altre società o enti. Peraltro esso vale per le società con socio pubblico di maggioranza o titolare di società capogruppo. Siamo quindi in presenza di società miste, nelle quali la presenza di capitali privati dovrebbe, in via di massima, essere giustificata dalla ricerca del profitto.

\subsection{Un secondo argomento}

Un secondo argomento a favore del ricorso alla spa potrebbe essere quello del profitto. E' un tema da considerare da angoli diversi, sia nel caso di società a piena proprietà pubblica, sia di società miste. In partenza, la presenza di profitto, o comunque di un saldo positivo tra ricavi e costi, potrebbe essere intesa come prova di efficienza. Le imprese private, in generale, se realizzano profitto sono considerate efficienti, se non creano profitto non efficienti: dato che le imprese private sono generalmente costituite in forma di spa, la spa sarebbe la veste migliore anche per la gestione della cosa pubblica.

Questo ragionamento, che oggi è moneta corrente, si inquadra a fatica nell'impostazione tradizionale dell'economia pubblica italiana (o scienza delle finanze) portata ad escludere la leva del profitto tra gli interessi pubblici che giustificano l'impresa pubblica.

"Vuolsi ... notare come il mero scopo di ottenere un reddito fiscale non sia una ragione sufficiente ed accettabile per legittimare l'assunzione di un'impresa da parte dell'ente collettivo. Se, invero, il desiderio di reddito a prò dell'erario pubblico è il solo movente, noi non siamo più nel campo dell'impresa pubblica, ma del demanio fiscale puro, senza nemmeno quei vantaggi incidentali pubblici che si sono osservati nel caso delle foreste; non c'è più ragione di fissare prezzi pubblici, anzi si devono fissare quei medesimi prezzi che vigono sul mercato". ${ }^{18}$

18 Einaudi L., Corso di scienza delle finanze. Edizione della Rivista "La riforma sociale", Terza edizione, Torino, 1916, p. 55. 
Inoltre, legare il tema dei profitti al tipo di proprietà è semplificazione accettabile per quanto riguarda l'an, ma non il quantum. Infatti, la misura dei profitti dipende poco dalla proprietà e molto dal grado di concorrenza del mercato in cui opera l'impresa, (se privata) dal grado di contendibilità del controllo azionario, dalle caratteristiche della regolamentazione dei processi e dei prodotti.

Relativamente ai settori di cui ci occupiamo, è tuttavia subito evidente che le spa strumentali o comunque tutte le società pubbliche, che hanno ricavi totalmente dipendenti dai contratti di servizio, sono lontane da queste considerazioni. Infatti, per far apparire il profitto nel bilancio della spa può bastare una partita di giro nel bilancio dell'ente proprietario.

In concreto, comunque, non si registrano grandi profitti tra le società strumentali. Nell'indagine sul capitalismo municipale di Scarpa ed altri (relativa ad un campione di 711 partecipate da enti pubblici), la categoria di spa della categoria "servizi" - servizi diversi dalle utility e dai trasporti e categoria che dovrebbe includere le società che in questa nota definiamo strumentali - registra, unica insieme ai trasporti, un utile per addetto negativo. ${ }^{19}$ Nel lavoro di Scarpa si nota che l'assenza generalizzata di profitti in questo genere di spa pubbliche suscita "interrogativi particolarmente pesanti", perché esse opererebbero "in settori di attività estranei all'ambito tradizionalmente affidato all'intervento pubblico" ${ }^{20}$ In realtà, questi settori dovrebbero essere meglio individuati perché, se corrispondessero alle attività che qui definiamo strumentali, l'assenza di profitti sarebbe fisiologica.

Sempre come dato di fatto, dall'indagine della Corte dei Conti risulta che al 2007 il $63 \%$ delle partecipate locali in perdita rientravano nella categoria delle società strumentali e delle "altre"( ). ${ }^{21}$ Rispetto ai totali per categoria, quelle in perdita erano al 38\% nel campo dei servizi e al $40 \%$ nel campo delle strumentali e altre.

Le società strumentali, dunque, sono d'ordinario diverse dalle società private perché non realizzano e non sono richieste di realizzare

19 Scarpa C., Bianchi P., Bertolotti B. \& Pellizzola L., Comuni Spa. Il capitalismo municipale in Italia, il Mulino, Bologna, 2009, pp. 134-136.

${ }^{20}$ Ivi, p. 136.

${ }^{21}$ Corte dei Conti, 2010. Indagine sul fenomeno delle partecipazioni in società ed altri organismi da parte di Comuni e Province, Deliberazione n.14/szaut/2010/frg, passim. 
profitti, ma "senza profitti" non significa nonprofit. Generalmente, le società non profit sono senza profitti distribuiti ma ad alto tasso di imprenditorialità; le pubbliche strumentali sono senza profitti tout court e a basso indice di imprenditorialità.

"Given the limits to pecuniary reward in the nonprofit sector, it is easy to assume that the supply of nonprofit entrepreneurial talent is limited. Non-pecuniary motivations however are common and potent, and entrepreneurial ventures abound in the nonprofit sector. These individuals are probably different from those pertaning to business entrepreneurs. These differences characterize also the management. In contrast to private corporations where boards of directors are responsible to and elected by shareholders, board members of nonprofit organizations are usually elected or appointed by a small group of activists in the organization. There are no shareholders that must be satisfied. In contrast to governmental enterprises, there are no general political interest, that is voters, that must be satisfied, except powerful business interests with large contributions. In a sense, the nonprofit boards are responsible to no one but themselves and a small leadership within the organization and influential donors with political or ideological agenda".22

Nelle spa non strumentali le realizzazione di profitti è auspicabile? Dipende.

Anzitutto, va ricordato che, secondo l'ordinamento comunitario, gli enti pubblici possono avviare e mantenere iniziative imprenditoriali, purché non venga lesa la concorrenza. Non sono ammessi aiuti palesi o mascherati verso la società. Tuttavia, anche senza aiuti, nei fatti è privilegiata un'impresa che si confronti con altre imprese su un mercato concorrenziale e che, a differenza delle altre, non abbia un vincolo di profitto. Perché mai un soggetto pubblico dovrebbe costituire un impresa e quindi una spa escludendo il profitto? Potrebbe essere mosso dall'intenzione di rendere un determinato mercato più articolato e competitivo. Anche in questo caso, però, l'intervento di un nuovo competitore, pubblico, dovrebbe ottenere una riduzione del livello dei profitti, ma non una loro scomparsa.

L'ente proprietario potrebbe essere concentrato sull'interesse dei clienti o utenti della spa e potrebbe chiedere a questa di applicare prezzi o tariffe inferiori a quelle che determinano i profitti. A parte che,

22 Auteri M., The entrepreneurial establishment of a non profit organization, Public Organization Review: A Global Journal, 2003, 3:184-185. 
come detto sopra, in questo scenario le condizioni di concorrenza alla lunga verrebbero meno, non c'è ente territoriale che, più o meno esplicitamente, non bilanci gli interessi degli utenti con quello dei contribuenti. Un esempio per chiarire. I Comuni di Brescia e Milano non applicano l'addizionale Irpef. Questa invece è applicata dalla generalità, o quasi, dei Comuni che, a differenza di Brescia e Milano, non possono contare sui profitti delle ex municipalizzate del gas. E dunque, è preferibile addizionare la tassazione dei redditi e ridurre la tariffe o, all'opposto, mantenere alte le tariffe energetiche (sui consumi domestici) e non inasprire l'Irpef? Quanto meno è un quesito appropriato.

Complessivamente, fuori dal campo delle spa strumentali, il profitto dovrebbe essere considerato un requisito normale delle spa pubbliche operanti in un mercato regolamentato in concorrenza (per il mercato) con competitori pubblici o privati.

Quanto discusso sopra vale per le spa totalmente pubbliche. Per le miste, comprese le quotate, può essere diverso, perché il mantenimento di un livello positivo di profittabilità è normalmente condizione per l'apporto di capitali privati. Teoricamente, inoltre, le quotazioni borsistiche offrirebbero una spia costante dell'efficienza nella conduzione dell'impresa. Nondimeno anche nelle miste, non ad azionariato diffuso, ma con singoli o pochi partner societari, il profitto può non essere l'unico movente del socio privato. Per così dire, anche per il socio privato la spa può essere strumentale. Per esempio, un fabbricante privato di tubazioni e di pompe potrebbe partecipare ad una spa di gestione del ciclo integrato dell'acqua non per il profitto derivante da tale gestione, ma per garantire un cliente alla propria produzione industriale. Un caso simile è non solo lecito ma frequente nel contesto totalmente privato. In un contesto pubblico/privato la questione può porsi diversamente perché, se non altro, la normativa sulle forniture pagate con capitali pubblici può rendere vana l'ipotesi che il socio produttore di un determinato bene o servizio lo venda in esclusiva alla spa mista partecipata.

\subsection{Un terzo argomento}

Un terzo argomento a favore della costituzione di spa pubbliche locali è quello della ricerca delle giuste dimensioni o, in alcuni casi (vedi ciclo integrato dell'acqua), anche delle economie di varietà. In Italia abbiamo 8.101 Comuni, dei quali 5.720 (70,6\% del totale) con meno di 5.000 abitanti. Tali Comuni ospitano circa un sesto (10 milio- 
ni) di italiani, con una dimensione media di 1.815 abitanti. E' chiaro che la spa, nella sua logica basilare di e pluribus unum, può rappresentare una risposta a tale frazionamento. Di fatto, secondo i dati della Corte dei Conti, sotto i 5.000 abitanti si riscontra il 60\% delle partecipazioni, il $36 \%$ tra i 5.000 e i 100.000 abitanti e il $3 \%$ oltre i $100.000 .^{23}$ Si può quindi constatare una relazione molto pronunciata tra societarizzazione e piccole dimensioni. Non è però del tutto evidente quanto un così ampio numero di società sia effettivamente necessario per ridurre gli inconvenienti della piccola dimensione e della frammentazione.

Relativamente alle spa strumentali, sorge l'interrogativo se sia saggio che queste spa, diciamo unificanti o consortili, si occupino di una sola funzione. E' corretto operare con una spa per la riscossione dei tributi locali, con un'altra e diversa per la mobilità scolastica, e via esemplificando? Dato che i bacini d'utenza e le dimensioni ottime delle diverse funzioni non sempre coincidono, queste diverse spa potrebbero allineare partecipazioni azionarie non sempre sovrapponibili. Inoltre si può ritenere che i Comuni più piccoli, nonostante la dedizione di sindaci e amministratori, siano in maggiori difficoltà a seguire lo stato delle proprie partecipazioni e si può supporre, ovviamente, che siano proprio i Comuni di minori dimensioni ad avere bisogno di spa. Insomma, se le spa sono monotematiche si genera uno stato di complessità e probabilmente di confusione che è in contrasto con il supposto maggior pregio dei piccoli Comuni, che sarebbe il più diretto contatto tra amministrati e amministratori, da cui dovrebbero derivare grande trasparenza e forte responsabilità. In concreto il numero medio di partecipazioni in essere è 4 per i Comuni sotto i 5.000 abitanti, $5 / 6$ per i Comuni da 5.000 a 100.00 e $21 / 22$ sopra i $100.000 .{ }^{24}$ I dati sono però riferiti all'insieme delle partecipazioni (servizi pubblici, società strumentali e altre). Quale che sia la situazione attuale, come rimedio al sottodimensionamento comunale è preferibile che la spa strumentale intercomunale sia a largo spettro di attività. La manovra della scorsa estate impone ai Comuni tra i 30.000 e i 50.000 abitanti di contare su una sola società strumentale. Non è logicamente chiarissimo perché anche tra Comuni piccoli non si

23 Corte dei Conti, 2010. Indagine sul fenomeno delle partecipazioni in società ed altro organismi da parte di Comuni e Province, Deliberazione n. 14/szaut/2010/frg, p. 10.

${ }^{24}$ Ivi, p. 11. 
debba contare su di una sola società consortile strumentale (raggruppante Comuni con complessivamente più di 30.000 abitanti).

La soglia dei 30.000 abitanti dovrebbe avere qualche rilievo anche per il processo associativo dei Comuni di piccola taglia. Essenzialmente la situazione giuridica si presenta come segue. Per effetto dell'art. 14, c. 28, legge 122/2010, i Comuni sotto i 5.000 abitanti sono obbligati a esercitare in forma associata, attraverso Convenzione o Unione, le funzioni fondamentali "provvisoriamente individuate" dall'art. 21, c. 3 della legge 42/2009. Una parte di queste potrebbe essere erogata mediante spa che dovrebbe servire almeno 30.000 cittadini, mentre spetta alle Regioni individuare la dimensione ottimale e omogenea per lo svolgimento in forma associata delle medesime funzioni fondamentali. Potremo quindi avere piccoli Comuni facenti parte di una sola Convenzione, o di una sola Unione, ma soci di più società strumentali partecipate dai Comuni della Convenzione, o Unione, più altri Comuni fino a superare la soglia dei 30.000 abitanti. Sembra essere un buon contributo all'impazzimento della maionese istituzionale.

\section{COME NEL PRIVATO}

Infine, a favore della costituzione di spa pubbliche locali milita uno dei principali argomenti della dottrina amministrativa nota come new public management. Questa, in una battuta, incita a "fare come nel privato", essendo però scontato che, come già sottolineato, una spa pubblica non avverte gli incentivi ad operare efficientemente che, si suppone, ha una spa privata in ragione della trasferibilità delle quote. La spa pubblica per definizione è non contendibile. La spa privata lo dovrebbe essere, ma nei fatti spesso anch'essa è un castello inespugnabile. Ne consegue che quel tanto che il privato può suggerire al pubblico, nel perseguimento dell'efficienza, non deriva dalla mutabilità del possesso azionario.

Non deriva, salvo eccezioni, neppure dalla possibilità di fallire, $\mathrm{o}$ comunque di chiudere, in caso di cattiva gestione. Nel privato, secondo la dottrina economica mainstream, l'ipotesi del fallimento riduce l'azzardo morale cui possono indulgere manager e dirigenti delle imprese, determinando una trascurata cura di costi e ricavi. Nel pubblico, invece, si suppone che l'azionista non accetti la chiusura e che, quindi, sia disposto a soccorrere (bail out) la società con ripetuti apporti di capitale a ripiano delle perdite. 
Il new public management, essenzialmente, suggerisce il superamento di regole e controlli sui procedimenti e prescrive il massimo dell'attenzione sui risultati. In poche parole, la ricetta è autonomia e responsabilità.

\subsection{Autonomia}

Il 30 giugno scorso la Camera dei Deputati ha approvato la "Carta delle autonomie", che è ora in discussione al Senato (Atti n. 2259). Il disegno di legge si occupa dei controlli sulle società partecipate nei seguenti termini: "1. L'ente locale definisce, secondo la propria autonomia organizzativa, un sistema di controlli sulle società partecipate ...2. ... l'amministrazione definisce preventivamente ... gli obiettivi gestionali a cui deve tendere la società partecipata, secondo standard qualitativi e quantitativi, e organizza un idoneo sistema informativo finalizzato a rilevare i rapporti finanziari tra l'ente proprietario e la società, la situazione contabile, gestionale e organizzativa delle società, i contratti di servizio, la qualità dei servizi, il rispetto delle norme di legge sui vincoli di finanza pubblica. 3 ... l'ente locale effettua il monitoraggio periodico sull'andamento delle società partecipate, analizza gli scostamenti rispetto agli obiettivi assegnati e individua le opportune azioni correttive, anche in riferimento a possibili squilibri economico-finanziari rilevanti per il bilancio dell'ente". (art. 24, a proposito dell'art. 147 ter del nuovo Tuel).

A fronte di un testo siffatto, suggerirei un emendamento riassuntivo e sostitutivo di questo tipo: "I conti delle aziende sono sottoposti ..., con speciale relazione, alle deliberazioni del consiglio comunale. Detti conti saranno depositati nella segreteria comunale in modo che tutti gli elettori possano prenderne visione". E' un virgolettato che rileggo nel testo unico sulla municipalizzazione del 1925 (art. 16, che riprende la legge 103/1903 e il regio decreto 3047/1923). Che le aziende pubbliche vadano controllate in un quadro di trasparenza non sorprende più di tanto.

\subsection{Responsabilità}

In Cina, come è noto, sono in atto da due o tre decenni profondi rivolgimenti economici. Le imprese, prima della più recente apertura ai capitali privati anche stranieri e alle quotazioni borsistiche, sono state riorganizzate dal modello di programmazione più o meno socialista, 
con forte controllo dal Partito comunista, in strutture giuridiche simili alle nostre società per azioni o cooperative, ma sempre a totale proprietà pubblica. Si è dunque verificato un colossale fenomeno di societarizzazione che ha incuriosito i ricercatori, soprattutto cinesi. Alcuni tra questi hanno individuato i meriti della societarizzazione, a costante proprietà pubblica, in un elemento caratteristico della proprietà privata diverso dai due argomenti della circolazione delle quote e del fallimento, precedentemente più volte ricordati. ${ }^{25}$

Sul piano concettuale tale specifico fattore disciplinante della proprietà privata viene individuato nel contenuto di un "teorema di Alchian", esposto in una pubblicazione americana del 1977 che gli autori ritengono seminale. ${ }^{26}$ Piace però notare che uno scritto analogo di Alchian fu pubblicato da Il Politico, la rivista della Facoltà di Scienze politiche di Pavia, già nel 1965..

Il teorema di Alchian, in sostanza, afferma che tra proprietà privata e pubblica la differenza è da ricercare nella maggior concentrazione della responsabilità. "Nel pubblico la responsabilità di ogni scelta è spinta con minor forza che nel privato in capo al decisore". ${ }^{28}$ Un esempio, tratto dallo stesso testo, aiuta a comprendere il concetto. Pensiamo al servizio di raccolta dei rifiuti urbani, un servizio che allo stesso tempo ha natura di bene privato e di bene pubblico. Se il servizio non funzio$\mathrm{na}$, in un contesto pubblico al massimo il responsabile non viene rieletto. In un conteso privato il costo di scelte sbagliate è più elevato. Il decisore, ovvero l'imprenditore in questo caso, può anche perdere l'intero capitale investito. E' chiaro che, per arrivare al cuore del discorso, si ricorre ad un'astrazione tale, in questo caso, da rasentare la favola perché, in ambito privato, raramente i decisori pagano per i propri errori, soprattutto se sono a capo di organismi di vaste proporzioni. Si veda, da ultimo, quanto accaduto con la presente crisi, prima finanziaria ora reale, con la diffusione dei mutui subprime, dei derivati e di altre perniciose sofisticazioni finanziarie.

${ }^{25}$ Lixin Colin X., Tian Z. \& Yi-min L., Politician control, agency problems and ownership reform. Evidence from China, Economics of transition, 2005, 13, pp. 1-24.

26 Alchian A.A., Some economics of property rights. Economic forces at work. Liberty Press, Indianapolis, 1977. 816-830

27 Alchian A.A., Some economics of property rights. Il Politico, 1965, XXX, pp.

${ }^{28}$ Ivi, p. 827. 
Tuttavia, anche in presenza di proprietà totalmente pubblica, si può recuperare almeno una parte dell'attitudine a concentrare la responsabilità che sarebbe insita nella proprietà privata? In Cina sembra sia stato possibile essenzialmente focalizzando meglio, rispetto al passato, il ruolo dei manager. Infatti, le imprese prima fortemente dipendenti dalla burocrazia statale e dal potere politico sono state ristrutturate con un consiglio di amministrazione (board of directors) che, senza assemblea dei soci nel caso di proprietà totalmente pubbli$\mathrm{ca}$, nelle imprese corporatized è stato ed è libero (o comunque più libero che nelle imprese noncorporatized) di assumere e licenziare i manager, provvedendo alla remunerazione di costoro secondo contratti incentivanti. Soprattutto grazie a questo accorgimento, nel corso degli anni '90 del secolo scorso, la societarizzazione delle imprese cinesi ha comportato vantaggi in termini di profittabilità dei capitali impegnati, di efficienza (misurata in termini di output o di fatturato per addetto) e di investimenti. ${ }^{29}$

Dall'insieme di queste considerazioni, parte teoriche e parte empiriche, si è indotti a concludere che la gestione, non burocratica e non ingessata, del personale sia di fondamentale importanza e che, conseguentemente, sia necessario fare spazio a contratti incentivanti (probabilmente non solo nei confronti dei manager), a premi e a disincentivi, nonché a sistemi di valutazione ex post delle attività di individui e reparti. Se tutto questo è plausibile, non è però scontato che, nell'ambito del settore pubblico locale, per ottenere un'organizzazione del lavoro maggiormente flessibile e produttiva sia necessario deliberare l'avvio di una spa o l'acquisto di una partecipazione. Nel contesto istituzionale italiano, in particolare, vige, e dovrebbe produrre effetti, il D.lgs 150/2009 di attuazione della legge 15/2009 "in materia di ottimizzazione della produttività del lavoro pubblico e di efficienza e trasparenza delle pubbliche amministrazioni". Si tratta di un testo legislativo forte di 74 articoli, alcuni lunghi pagine e pagine, fitte fitte. Sono articoli svolti soprattutto attorno a concetti quali obiettivi, indicatori, performances, misurazione e valutazione.

Cosicché si può per esempio leggere che "La misurazione e la

${ }^{29}$ Aivazian V.A., Yin G. e Jiaping Q., Can corporatization improve the performance of state-owned enterprises even without privatization?, Journal of Corporate Finance, 2005, 11, pp. 791-808. 
valutazione della performance individuale dei dirigenti e del personale responsabile di una unità organizzativa in posizione di autonomia e responsabilità sono collegate: a) agli indicatori di performance relativi all'ambito organizzativo di diretta responsabilità; b) al raggiungimento di specifici obiettivi individuali; ..." (art. 9). Più avanti si stabilisce che "Ogni amministrazione ha l'obbligo di pubblicare sul proprio sito istituzionale in apposita sezione di facile accesso e consultazione: ...c) l'ammontare complessivo dei premi collegati alla performance stanziati e l'ammontare dei premi effettivamente distribuiti; d) l'analisi dei dati relativi al grado di differenziazione nell'utilizzo della premiabilità sia per i dirigenti, sia per i dipendenti; ... g) le retribuzioni dei dirigenti, con specifica evidenza sulle componenti variabili delle retribuzioni e delle componenti legate alla valutazione di risultato" (art. 11, c. 8). Infine, per tacer d'altro in questa sede, in tema di principi generali del lavoro pubblico si stabilisce una "disciplina della dirigenza pubblica" tale da "conseguire la migliore organizzazione del lavoro e assicurare il progressivo miglioramento delle prestazioni erogate al pubblico, utilizzando anche i criteri di gestione e di valutazione del settore privato ..." (art. 37).

\section{IL PROBLEMA DI AGENZIA}

Nell'esempio cinese, la societarizzazione, rispetto alla precedente forma di organizzazione delle imprese, ha rappresentato un progresso essenzialmente perché, con essa, è stato ridotto il "costo politico", cioè il danno prodotto dalle ingerenze della politica nella produzione. Si sono però manifestate altre criticità. E' rimasto un generale problema di soft budget constraint (salvataggio sistematico delle imprese in difficoltà da parte dell'azionista Stato) e, facendo largo al potere decisionale dei manager, è inevitabilmente sorto il problema di agenzia ${ }^{30}$. Questo è costituito dall'incerta e parziale coincidenza della condotta dei manager, cioè dei decisori effettivi all'interno delle imprese, con gli interessi degli azionisti. Nulla di nuovo agli occhi dell'osservatore occidentale: il famoso lavoro di Berle e Means sulla separazione tra proprietà e con-

30 Lixin Colin X., Tian Z. \& Yi-min L. Politician control, agency problems and ownership reform. Evidence from China, Economics of transition, 2005, 13, pp. 1-24. 
trollo risale agli anni ' 30 del '900. ${ }^{31}$ Ad attenuare tali nuovi problemi è sembrata necessaria la ricerca sistematica del profitto (tra l'altro abolendo i sistemi di golden share) ed è quindi sembrato necessario l'ulteriore passo costituito dall'apertura al capitale privato, talché la maggior parte delle imprese che negli anni ' 90 sono state societarizzate è ora una società mista. Indipendentemente da questa evoluzione, l'insegnamento che si può trarre dall'esperienza in Cina può essere di qualche significato anche per noi, sebbene il fenomeno della societarizzazione si sia sviluppato essenzialmente in una platea di imprese industriali, per le quali è supposto l'orientamento al profitto ed è relativamente agevole quantificare e valutare il prodotto.

Ma la misura del prodotto è cruciale anche al di fuori del settore industriale. In sostanza, si può verificare se e come e quanto il passaggio alla forma societaria comporta vantaggi rispetto alla gestione interna alla pubblica amministrazione? Il quesito vale per ogni singolo caso e per l'intero comparto delle società partecipate dall'ente pubblico, con particolare riguardo alle strumentali. Come abbiamo già visto, il solo test dell'esistenza di un profitto è inapplicabile o inadeguato. Nella (scarna) letteratura internazionale il tema è trattato contrapponendo i risultati post societarizzazione ai dati fatti registrare dall'agenzia pubblica, prima della sua trasformazione in società (corporation o company). Il termine "agenzia pubblica" (agency o government department o government unit) è attribuito ad un campo assai ampio di organismi. Nel caso nostro, specificamente delle società comunali strumentali, dovrebbe essere riferibile anche ad un assessorato o ad una sua parte in modo da rendere percorribile il confronto, per esempio, sulla refezione scolastica a Milano, prima e dopo l'avvento della specifica società. In altri termini, dovremmo saper confrontare il servizio di ristorazione operato, come a Milano, mediante spa con il medesimo servizio garantito in città simili mediante i consueti apparati amministrativi. Naturalmente l'indagine dovrebbe assumere i confronti con le esternalizzazioni vere (appalti a imprese private). Così impostata, si tratterebbe di un ricerca simile a quella, ormai enorme, sull'operatività di imprese pubbliche e imprese private (operanti nel medesimo settore, l'acqua, l'energia elettrica, ecc.). La comparazione pubblico/privato, tuttavia, si concentra sulla determinazione del livello di profittabilità (il roe, return on equity e il roa, return on assets sono moneta

31 Berle A. \& G. Means, The Modern Corporation Private Propriety, 1932 (2 ${ }^{\text {nd }}$ edn Hearcourt, Brace and World, New York, 1967). 
corrente in simili studi) delle diverse imprese e si avvale di informazioni quantitative già organizzate omogeneamente nei diversi bilanci societari. Per entrambi questi aspetti, l'analisi della societarizzazione si differenzia da quella sulla privatizzazione. In particolare il problema della confrontabilità dei dati si presenta come assai serio.

Ammesso di poter identificare l'agenzia trasformata in spa e, beninteso, i relativi dati, la letteratura identifica i seguenti parametri di controllo dei guadagni di efficienza derivabili dalla societarizzazione:

a. rispetto all'agenzia governativa, la spa pubblica determina un aumento dell'output;

b. la spa aumenta gli introiti;

c. la spa garantisce una miglior copertura dei ricavi sui costi;

d. la spa migliora la produttività (l'impiego di input a parità di output);

e. la spa perfeziona l'organizzazione e la produttività del lavoro: output o vendite per addetto. ${ }^{32}$

Elencando questi indici non si fa altro che tracciare una programma di ricerca empirica, che peraltro si prospetta di non facile svolgimento data la povertà dei dati disponibili. Tuttavia, anche la sola semplice elencazione dei fattori da controllare indica un'ulteriore condizione e un definitivo fattore di successo della trasformazione in spa. Essenzialmente la societarizzazione di una unità amministrativa ha senso soltanto in presenza di attività con esiti quantificabili e, probabilmente, senza soverchie graduazioni qualitative. Quando, invece, più che conteggiare prodotti si possono, e non è poco, elencare obiettivi e descrivere perfomances individuali, o di reparto o di team, i vantaggi della spa sbiadiscono alquanto.

In particolare, le spa (come ne esistono per esempio in Lombardia) incaricate unicamente di predisporre appalti e gare, nonché di stipulare contratti di fornitura costituiscono il caso più equivoco e meno giustificabile di capitalismo comunale o provinciale o regionale.

\section{PER CONCLUDERE}

Le società pubbliche locali presentate inizialmente come soluzioni di mercato, con il loro proliferare hanno finito per ostacolare e ridur-

32 Cfr. per esempio, Bilodeau N., Laurin C. \& Vining A., Choice of organizational form makes a real difference: The impact of corporatization on government agencies in Canada. Journal of Public Administration Research and Theory, 2006, 1, pp. 119-147. 
re il mercato. Tuttavia, i danni prodotti al mercato sono forse inferiori a quelli prodotti allo Stato, alla sua logica e alla sua essenza.

E' diffusa l'impressione, vuoi la convinzione, che il nostro sistema di governo e di amministrazione pubblica sia eccessivamente complesso. Abbiamo quattro livelli base: Stato, Regioni, Province e Comuni. Non abbiamo, invece, un livello di governo di cui ci sarebbe molto bisogno, come dimostrano le vicende economiche degli ultimi anni. Il livello di governo europeo esistente è soltanto un abbozzo, largamente sottodotato di poteri effettivi. Inoltre, nonostante sia previsto in Costituzione (art. 114), non abbiamo ancora il livello governativo della Città metropolitana, della quale ci sarebbe urgente bisogno. All'interno, ai quattro livelli base, si aggiungono Comunità montane (spesso sois disant montane) e Unioni comunali e altre svariate forme di gestione associata dei servizi, gestione resa necessaria o soltanto utile dato il forte sottodimensionamento della maggior parte dei Comuni. A produrre servizi pubblici o attività strumentali alle funzioni pubbliche, infine, sono chiamate, con mandati più o meno precisi e imperativi, anche le quasi seimila entità societarie e simili censite dall'indagine della Corte dei Conti più volte citata. Queste, se in qualche caso offrono un contributo a superare gli inconvenienti del sottodimensionamento soprattutto dei Comuni, nel loro complesso aggiungono complessità ad un quadro già abbastanza confuso e datato.

Un'entità che denominiamo azienda o impresa, giuridicamente vestita come spa, si intende ontologicamente soggetta agli stimoli e ai vincoli del mercato. Se il mercato latita, come ordinariamente avviene nei settori di maggiore interesse della pubblica amministrazione, essa si affloscia come un sacco vuoto. Per tenerla in piedi senza troppo nuocere, si è costretti ad introdurre una serie di supporti, nonché di vincoli di carattere pubblico, che creano una figura istituzionale ibrida. Anche lo storico modello della municipalizzata e l'attuale schema dell'azienda speciale introducono simulazioni aziendalistiche e privatistiche in ambiente pubblicistico. Rispetto a queste forme giuridico-organizzative anch'esse meticce, però, la società pubblica locale, considerando l'esperienza di questi ultimi quindici anni, sembra richiedere una serie di interventi legislativi di raccordo, integrazione e specializzazione molto maggiore. Inoltre, in rapporto a municipalizzate e aziende speciali, la società pubblica locale è soccombente al test della trasparenza. 